\title{
Reliable algorithms for computing minimal dynamic covers
}

\author{
Andras Varga \\ German Aerospace Center \\ DLR - Oberpfaffenhofen \\ Institute of Robotics and System Dynamics \\ D-82234 Wessling, Germany \\ Andras.Varga@dlr.de
}

\begin{abstract}
Minimal dimension dynamic covers play an important role in solving the structural synthesis problems of minimum order functional observers or fault detectors, or in computing minimal order inverses or minimal degree solutions of rational equations. We propose numerically reliable algorithms to compute two basic types of minimal dimension dynamic covers for a linear system. The proposed approach is based on a special controllability staircase condensed form of a structured pair $\left(A,\left[B_{1}, B_{2}\right]\right)$, which can be computed using exclusively orthogonal similarity transformations. Using such a condensed form minimal dimension covers and corresponding feedback/feedforward matrices can be easily computed. The overall algorithm has a low computational complexity and is provably numerically reliable.
\end{abstract}

\section{INTRODUCTION}

Our main motivation to address the computational aspects of determining minimal dimension dynamic covers is the following concrete problem encountered in the design of least order fault detectors [14], [13]: for given proper rational matrices $X_{1}(\lambda)$ and $X_{2}(\lambda)$ with the same row dimensions, determine appropriate proper $Y(\lambda)$ such that $X_{1}(\lambda) Y(\lambda)+X_{2}(\lambda)$ has the least possible McMillan degree. Let $\left(A,\left[B_{1}, B_{2}\right], C,\left[D_{1}, D_{2}\right]\right)$ be a minimal state-space realization of $\left[X_{1}(\lambda) X_{2}(\lambda)\right]$ satisfying

$$
\left[X_{1}(\lambda) X_{2}(\lambda)\right]=C(\lambda I-A)^{-1}\left[\begin{array}{ll}
B_{1} & B_{2}
\end{array}\right]+\left[\begin{array}{ll}
D_{1} & D_{2}
\end{array}\right]
$$

It was shown in [6] that under certain conditions (i.e., maximally observable realization), determining the appropriate $Y(\lambda)$ can be recast as a problem to compute a state feedback matrix $F$ and a feedforward matrix $G$ to achieve the cancellation of a maximum number of uncontrollable poles of the transfer-function matrix

$$
R(\lambda)=C_{F}\left(\lambda I-A_{F}\right)^{-1} B_{G}+D_{G}
$$

where $A_{F}:=A+B_{1} F, B_{G}=B_{1} G+B_{2}, C_{F}=C+D_{1} F$, and $D_{G}=D_{1} G+D_{2}$. Different instances of this problem appear in solving various structural synthesis problems, as for example, the design of minimum order functional observers [4], determining minimal order inverses [1] or computation of minimal degree solutions of rational equations [6]. In all these cases, the proposed solution procedures reformulate these problems as minimum dynamic cover problems, which can be solved using the "standard" method of [17] relying on subspace manipulation techniques employed in the geometric theory of linear systems [16].
Turning such an approach into an efficient and numerically reliable algorithm is not straightforward. A first difficulty when performing the computations in [17] (see also [4]), is the need to reduce the system matrices to a special canonical form which exhibits the structural information necessary to solve the problem. However, this canonical form can only be computed by using non-orthogonal transformations resulted from a special basis selection procedure and is based on rank decisions involving successive powers of $A$. This approach is basically equivalent to test controllability by checking the rank of the explicitly constructed controllability matrix, which is known to be a notoriously ill-conditioned computational problem [7]. The second aspect is the possibly high computational complexity. Although the basis selection algorithms can be turned into reliable numerical computations using, for example, the technique for the calculus with subspaces proposed in [5], the resulting algorithm has a worst-case computational complexity of $O\left(n^{4}\right)$, where $n$ is the order of $A$. This high complexity is the result of the need to accumulate and apply at each reduction step the orthogonal transformations performed at one step (e.g., the left and right orthogonal transformations to compute singular value decompositions). Thus it appears that there is no satisfactory algorithm to compute minimal dynamic covers and the associated feedback/feedforward matrices.

In this paper we propose a numerically reliable and computationally efficient approach to compute a feedback matrix $F$ and a possibly nonzero feedforward matrix $G$ to achieve the desired cancellation of maximum number of uncontrollable poles in (1). We solve the problems of determining both $F$ and $G$ or only $F$ which lead to cancellation of maximum number of uncontrollable poles. Solving these problems involves to compute bases for subspaces representing minimal dimension dynamic covers of Type II and Type I, respectively (see [4]). The main computational ingredient in these computations is bringing the system matrices into special condensed forms which exhibit the structural information necessary to solve the problem. For the matrices in these condensed forms the computation of appropriate $F$ and $G$ is a simple, almost trivial task.

The algorithm to compute the condensed form has two stages: (1) an orthogonal reduction of the structured pair $\left(A,\left[B_{1}, B_{2}\right]\right)$ to a special controllability staircase form; and (2) a non-orthogonal transformation to zero addition- 
ally a minimum number of elements followed by special row/column block permutations. The orthogonal reduction part is based on employing techniques similar to that used in the controllability staircase form algorithms for standard systems [9], [8]. This part involves many rank decisions which can be computed by using reliable techniques (e.g., singular values based rank evaluations). The non-orthogonal part of the reduction does not involve any rank computations and is performed to allow an easy computation of appropriate feedback/feedforward matrices. The overall algorithm has a low computational complexity and is provably numerically reliable.

In the last part we also address shortly the solution of minimum cover problems with stability constraints. In the case the minimum cover problem with stabilization is solvable, we propose a reliable computational solution to this problem by exploiting the existing parametric freedom in the cover determination problem.

\section{COMPUTATION OF TYPE II MINIMAL DYNAMIC COVERS}

The computational problem which we solve is the following: given the pair $(A, B)$ with $A \in \mathbb{R}^{n \times n}, B \in \mathbb{R}^{n \times m}$, and $B$ partitioned as $B=\left[\begin{array}{ll}B_{1} & B_{2}\end{array}\right]$ with $B_{1} \in \mathbb{R}^{n \times m_{1}}$, $B_{2} \in \mathbb{R}^{n \times m_{2}}$, determine the matrices $F$ and $G$ such that the pair $\left(A+B_{1} F, B_{1} G+B_{2}\right)$ has maximal number of uncontrollable eigenvalues. This problem is essentially equivalent [6] to compute a subspace $\mathcal{V}$ having least possible dimension satisfying

$$
\begin{array}{rll}
\left(A+B_{1} F\right) \mathcal{V} & \subset & \mathcal{V} \\
\operatorname{span}\left(B_{1} G+B_{2}\right) & \subset & \mathcal{V}
\end{array}
$$

If we denote $\mathcal{B}_{1}=\operatorname{span} B_{1}$ and $\mathcal{B}_{2}=\operatorname{span} B_{2}$, then the above conditions can be rewritten also as conditions defining a Type II dynamic cover [2], [4]

$$
\begin{array}{rll}
A \mathcal{V} & \subset & \mathcal{V}+\mathcal{B}_{1} \\
\mathcal{B}_{2} & \subset & \mathcal{V}+\mathcal{B}_{1}
\end{array}
$$

The computation of the minimal dynamic covers relies on the reduction of the pair $\left(A,\left[B_{1}, B_{2}\right]\right)$ to a particular condensed form, for which the solution of the problem is simple. This reduction is performed in two stages. The first stage is an orthogonal reduction which represents a particular instance of the controllability staircase procedure of [9], [8] applied to the pair $\left(A,\left[B_{1}, B_{2}\right]\right)$. This procedure can be seen as an orthogonal variant of the basis selection approach of [4] and therefore will be useful to construct both Type II and Type I minimal covers. In the second stage, additional zero blocks are generated in the reduced matrices using non-orthogonal transformations and by applying appropriate feedback and feedforward matrices. From the resulting overall transformation matrix, a basis for the minimum dynamic cover can be easily obtained. In what follows we present in detail these two stages.

\section{Stage I: Special Controllability Staircase Algorithm}

1. Set $j=1, r=0, k=2, \nu_{1}^{(0)}=m_{1}, \nu_{2}^{(0)}=m_{2}, A^{(0)}=$ $A, B_{1}^{(0)}=B_{1}, B_{2}^{(0)}=B_{2}, Z=I_{n}$.

2. Compute the orthogonal matrix $U_{1}$ to compress the matrix $B_{1}^{(j-1)} \in \mathbb{R}^{(n-r) \times \nu_{1}^{(j-1)}}$ to a full row rank matrix

$$
U_{1}^{T} B_{1}^{(j-1)}:=\left[\begin{array}{c}
A_{k-1, k-3} \\
0 \\
\nu_{1}^{(j-1)}
\end{array}\right] \rho_{1}^{(j)}
$$

3. Compute $U_{1}^{T} B_{2}^{(j-1)}$ and partition it in the form

$$
U_{1}^{T} B_{2}^{(j-1)}:=\left[\begin{array}{c}
A_{k-1, k-2} \\
X \\
\nu_{2}^{(j-1)}
\end{array}\right] \nu_{1}^{(j)}
$$

4. Compute the orthogonal matrix $U_{2}$ to compress the matrix $X \in \mathbb{R}^{\left(n-r-\nu_{1}^{(j)}\right) \times \nu_{2}^{(j-1)}}$ to a full row rank matrix

$$
\left.U_{2}^{T} X:=\underset{c}{A_{k, k-2}} \begin{array}{c}
\nu_{2}^{(j)} \\
0 \\
\nu_{2}^{(j-1)}
\end{array}\right] \rho_{2}^{(j)}
$$

5. Compute $\operatorname{diag}\left(I, U_{2}^{T}\right) U_{1}^{T} A^{(j-1)} U_{1} \operatorname{diag}\left(I, U_{2}\right)$ and partition it in the form

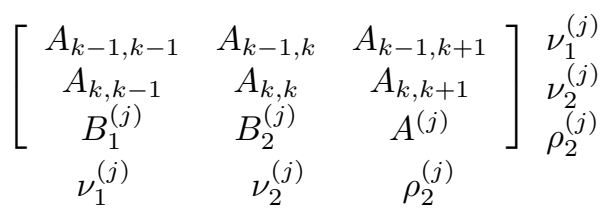

6. Compute for $i=1, \ldots, k-2$

$$
A_{i, k-1} U_{1} \operatorname{diag}\left(I, U_{2}\right):=\left[\begin{array}{llc}
A_{i, k-1} & A_{i, k} & A_{i, k+1}
\end{array}\right]
$$

7. $Z \leftarrow Z \operatorname{diag}\left(I_{r}, U_{1}\right) \operatorname{diag}\left(I_{r+\nu_{1}^{(j)}}, U_{2}\right)$.

8. $r \leftarrow r+\nu_{1}^{(j)}+\nu_{1}^{(j)}$; if $\rho_{2}^{(j)}=0$ then $\ell=j$ and Exit 1 .

9. If $\nu_{1}^{(j)}+\nu_{2}^{(j)}=0$ then $k \leftarrow k-2, \ell=j-1$, Exit 2;

else, $j \leftarrow j+1, k \leftarrow k+2$, and go to Step 2 .

At the end of this algorithm $\widehat{A}=Z^{T} A Z$ and $\widehat{B}=Z^{T} B$ have the following form

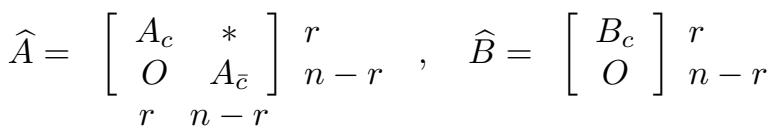

where the pair $\left(A_{c}, B_{c}\right)$ has only controllable eigenvalues, and $A_{\bar{c}}$ contains the uncontrollable eigenvalues of $A$. The 
pair $\left(A_{c}, B_{c}\right)$ is in the special staircase form with

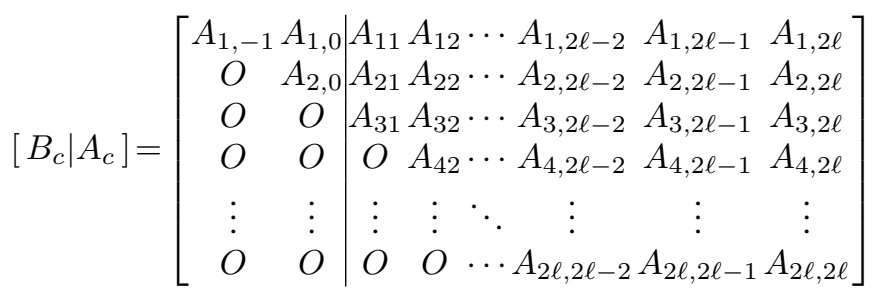

where $A_{2 j-1,2 j-3} \in \mathbb{R}^{\nu_{1}^{(j)} \times \nu_{1}^{(j-1)}}$ and $A_{2 j, 2 j-2} \in$ $\mathbb{R}_{2}^{\nu_{2}^{(j)} \times \nu_{2}^{(j-1)}}$ are full row rank matrices for $j=1, \ldots, \ell$.

To compute a Type II minimal cover, in the second reduction stage we use non-orthogonal upper triangular transformation matrices $U=\operatorname{diag}\left(U_{c}, I_{n-r}\right)$, respectively, to annihilate a minimum set of blocks in $A_{c}$. Assume $U_{c}$ has a supra-diagonal block structure identical to that of $A_{c}$. The following procedure performs the second reduction stage by exploiting the full row rank of submatrices $A_{2 k-1,2 k-3}$ to zero the blocks $A_{2 k-1,2 j}$, for $j=k-1, k, \ldots, \ell$ in row $2 k-1$ of $A_{c}$.

\section{Stage II: Special reduction for Type II Covers}

Set $U=I$.

for $k=\ell, \ell-1, \ldots, 2$

$$
\text { for } j=k-1, k, \ldots, \ell
$$

Compute $U_{2 k-3,2 j}$ such that

$$
A_{2 k-1,2 k-3} U_{2 k-3,2 j}+A_{2 k-1,2 j}=0
$$

For $i=1,2, \ldots, 2 k-1$ compute

$$
A_{i, 2 j} \leftarrow A_{i, 2 j}+A_{i, 2 k-3} U_{2 k-3,2 j}
$$

For $i=2 j-2, \ldots, 2 \ell$ compute

$$
A_{2 k-3, i} \leftarrow A_{2 k-3, i}-U_{2 k-3,2 j} A_{2 j, i}
$$

end

end

At the end of Stage II, the upper triangular matrix $U_{c}$ contains the accumulated non-orthogonal transformations performed in the reduction. Let $\widetilde{A}_{c}:=U_{c}^{-1} A_{c} U_{c}$, and $\widetilde{B}_{c}=$ $\left[\widetilde{B}_{c, 1} \widetilde{B}_{c, 2}\right]:=U_{c}^{-1} B_{c}$ be the system matrices resulted at the end of Stage II. Define also the feedback matrix $\widetilde{F}_{c} \in \mathbb{R}^{m_{1} \times r}$ partitioned column-wise compatibly with $\widetilde{A}_{c}$

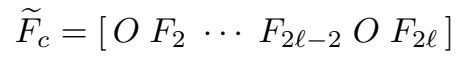

where $F_{2 j}$ are chosen such that $A_{1,-1} F_{2 j}+A_{1,2 j}=0$ for $j=1, \ldots, \ell$. Choose also $G$ such that $A_{1,-1} G+A_{1,0}=0$. These choices are always possible since $A_{1,-1}$ has full row rank.
With the computed $\widetilde{F}$ and $G$ we achieved that

$$
\left[\widetilde{B}_{c, 1} \widetilde{B}_{c, 1} G+\widetilde{B}_{c, 2}\right]=\left[\begin{array}{cc}
A_{1,-1} & O \\
O & A_{2,0} \\
O & O \\
O & O \\
\vdots & \vdots \\
O & O
\end{array}\right]
$$

$$
\widetilde{A}_{c}+\widetilde{B}_{c, 1} \widetilde{F}_{c}=\left[\begin{array}{cccccc}
\bar{A}_{11} & O & \cdots & O & \bar{A}_{1,2 \ell-1} & O \\
\bar{A}_{21} & \bar{A}_{22} & \cdots & \bar{A}_{2,2 \ell-2} & \bar{A}_{2,2 \ell-1} & \bar{A}_{2,2 \ell} \\
A_{31} & O & \cdots & O & \bar{A}_{3,2 \ell-1} & O \\
O & A_{42} & \cdots & \bar{A}_{4,2 \ell-2} & \bar{A}_{4,2 \ell-1} & \bar{A}_{4,2 \ell} \\
\vdots & \vdots & \ddots & \vdots & \vdots & \vdots \\
O & O & \cdots & A_{2 \ell, 2 \ell-2} & A_{2 \ell, 2 \ell-1} & A_{2 \ell, 2 \ell}
\end{array}\right]
$$

where the elements with bars have been modified in Stage II.

Consider now the permutation matrix defined by

$$
P^{T}=\left[\begin{array}{cc|c|cc|c}
O & I_{\nu_{2}^{(1)}} & \cdots & O & O & O \\
\hline \vdots & \vdots & \ddots & \vdots & \vdots & \vdots \\
\hline O & O & \cdots & O & I_{\nu_{2}^{(\ell)}} & O \\
\hline I_{\nu_{1}^{(1)}} & O & \cdots & O & O & O \\
\hline \vdots & \vdots & \ddots & \vdots & \vdots & \vdots \\
\hline O & O & \cdots & I_{\nu_{1}^{(\ell)}} & O & O \\
\hline O & O & \cdots & O & O & I_{n-r}
\end{array}\right]
$$

If we define $V=Z U P$ and $F=\left[\widetilde{F}_{c} O\right] V^{-1}$, then overall we achieved that

$$
\begin{gathered}
V^{-1}\left(B_{1} G+B_{2}\right)=\left[\begin{array}{c}
\breve{B}_{1} \\
O \\
O
\end{array}\right] \\
V^{-1}\left(A+B_{1} F\right) V=\left[\begin{array}{ccc}
\breve{A}_{1} & * & * \\
O & \breve{A}_{2} & * \\
O & O & A_{\bar{c}}
\end{array}\right],
\end{gathered}
$$

where

$$
\begin{gathered}
{\left[\breve{B}_{1} \mid \breve{A}_{1}\right]=\left[\begin{array}{c|cccc}
A_{2,0} & \bar{A}_{2,2} & \bar{A}_{2,4} & \ldots & \bar{A}_{2,2 \ell} \\
O & A_{4,2} & \bar{A}_{4,4} & \ldots & \bar{A}_{4,2 \ell} \\
\vdots & \vdots & \ddots & \ddots & \vdots \\
O & O & O & A_{2 \ell, 2 \ell-2} & A_{2 \ell, 2 \ell}
\end{array}\right]} \\
\breve{A}_{2}=\left[\begin{array}{cccc}
\bar{A}_{1,1} & \bar{A}_{1,3} & \ldots & \bar{A}_{1,2 \ell-1} \\
A_{3,1} & \bar{A}_{3,3} & \ldots & \bar{A}_{3,2 \ell-1} \\
\vdots & \ddots & \ddots & \vdots \\
O & O & A_{2 \ell-1,2 \ell-3} & \bar{A}_{2 \ell-1,2 \ell-1}
\end{array}\right]
\end{gathered}
$$

It follows by inspection that the pair $\left(\breve{A}_{1}, \breve{B}_{1}\right)$ is controllable. Thus, by the above choice of $F$ and $G$, we made $\sum_{i=1}^{\ell} \nu_{1}^{(i)}$ eigenvalues of $A+B_{1} F$ uncontrollable via 
$B_{2} G+B_{1}$, additionally to the $n-r$ uncontrollable original eigenvalues. The first $n_{c}=\sum_{i=1}^{\ell} \nu_{2}^{(i)}$ columns $V_{1}$ of $V$ satisfy

$$
A V_{1}=V_{1} \breve{A}_{1}-B_{1} F V_{1}, \quad B_{2}=V_{1} \breve{B}_{1}-B_{1} G
$$

and thus, according to (2), span a Type II dynamic cover of dimension $n_{c}$ for the pair $\left(A,\left[B_{1} B_{2}\right]\right)$. The following result can be shown using the results of [4]:

Theorem 1: The Type II dynamic cover $\mathcal{V}=\operatorname{span} V_{1}$ has minimum dimension.

\section{COMPUTATION OF TYPE I MINIMAL DYNAMIC COVERS}

The computational problem which we solve in this section is the following: given the pair $(A, B)$ with $A \in \mathbb{R}^{n \times n}$, $B \in \mathbb{R}^{n \times m}$, and $B$ partitioned as $B=\left[\begin{array}{ll}B_{1} & B_{2}\end{array}\right]$ with $B_{1} \in \mathbb{R}^{n \times m_{1}}, B_{2} \in \mathbb{R}^{n \times m_{2}}$, determine the matrix $F$ such that the pair $\left(A+B_{2} F, B_{1}\right)$ has maximal number of uncontrollable eigenvalues. This problem is essentially equivalent [17] to compute a subspace $\mathcal{V}$ having least possible dimension satisfying

$$
\begin{array}{rll}
\left(A+B_{2} F\right) \mathcal{V} & \subset & \mathcal{V} \\
\operatorname{span} B_{1} & \subset & \mathcal{V}
\end{array}
$$

These conditions can be rewritten also as conditions defining a Type I minimum dynamic cover [2], [4]

$$
\begin{array}{ll}
A \mathcal{V} & \subset \mathcal{V}+\mathcal{B}_{2} \\
\mathcal{B}_{1} & \subset \mathcal{V}
\end{array}
$$

To compute Type I covers, we perform first the Stage I orthogonal reduction on the pair $\left(A,\left[B_{1}, B_{2}\right]\right)$, as done in the previous section. However, at Stage II the non-orthogonal reduction annihilates a different set of blocks in $A_{c}$. The following procedure performs the second reduction stage by exploiting the full row rank of submatrices $A_{2 k, 2 k-2}$ to zero the blocks $A_{2 k, 2 j-1}$, for $j=k, k+1, \ldots, \ell$ in row $2 k$ of $A_{c}$.

\section{Stage II: Special reduction for Type I Covers}

Set $U=I$.

for $k=\ell, \ell-1, \ldots, 2$

$$
\text { for } j=k, k+1, \ldots, \ell
$$

Compute $U_{2 k-2,2 j-1}$ such that

$$
A_{2 k, 2 k-2} U_{2 k-2,2 j-1}+A_{2 k, 2 j-1}=0
$$

For $i=1,2, \ldots, 2 k$ compute

$$
A_{i, 2 j-1} \leftarrow A_{i, 2 j-1}+A_{i, 2 k-2} U_{2 k-2,2 j-1}
$$

For $i=2 j-3, \ldots, 2 \ell$ compute

$$
A_{2 k-2, i} \leftarrow A_{2 k-2, i}-U_{2 k-2,2 j-1} A_{2 j-1, i}
$$

end

\section{end}

Let $\widetilde{A}_{c}:=U_{c}^{-1} A_{c} U_{c}$ and $\widetilde{B}_{c}=\left[\widetilde{B}_{c, 1} \widetilde{B}_{c, 2}\right]:=U_{c}^{-1} B_{c}$ be the system matrices resulted at the end of Stage II. Define also the feedback matrix $\widetilde{F}_{c} \in \mathbb{R}^{m_{2} \times r}$ partitioned columnwise compatibly with $\widetilde{A}_{c}$

$$
\widetilde{F}_{c}=\left[\begin{array}{lllll}
F_{1} O F_{3} & \cdots & O F_{2 \ell-1} O
\end{array}\right]
$$

where $F_{2 j-1}$ are such that $A_{2,0} F_{2 j-1}+A_{2,2 j-1}=0$ for $j=1, \ldots, \ell$.

Consider now the permutation matrix defined by

$$
P^{T}=\left[\begin{array}{cc|c|cc|c}
I_{\nu_{1}^{(1)}} & O & \cdots & O & O & O \\
\hline \vdots & \vdots & \ddots & \vdots & \vdots & \vdots \\
\hline O & O & \cdots & I_{\nu_{1}^{(\ell)}} & O & O \\
\hline O & I_{\nu_{2}^{(1)}} & \cdots & O & O & O \\
\hline \vdots & \vdots & \ddots & \vdots & \vdots & \vdots \\
\hline O & O & \cdots & O & I_{\nu_{2}^{(\ell)}} & O \\
\hline O & O & \cdots & O & O & I_{n-r}
\end{array}\right]
$$

If we define $V=Z U P$ and $F=\left[\widetilde{F}_{c} O\right] V^{-1}$, then overall we achieved that

$$
\begin{aligned}
V^{-1} B_{1} & =\left[\begin{array}{c}
\breve{B}_{1} \\
O \\
O
\end{array}\right] \\
V^{-1}\left(A+B_{2} F\right) V & =\left[\begin{array}{ccc}
\breve{A}_{1} & * & * \\
O & \breve{A}_{2} & * \\
O & O & A_{\bar{c}}
\end{array}\right],
\end{aligned}
$$

where

$$
\begin{aligned}
{\left[\breve{B}_{1} \mid \breve{A}_{1}\right] } & =\left[\begin{array}{c|cccc}
A_{1,-1} & \bar{A}_{1,1} & \bar{A}_{1,3} & \ldots & \bar{A}_{1,2 \ell-1} \\
O & A_{3,1} & \bar{A}_{3,3} & \ldots & \bar{A}_{3,2 \ell-1} \\
\vdots & \vdots & \ddots & \ddots & \vdots \\
O & O & O & A_{2 \ell-1,2 \ell-3} & A_{2 \ell-1,2 \ell-1}
\end{array}\right] \\
\breve{A}_{2} & =\left[\begin{array}{ccccc}
\bar{A}_{2,2} & \bar{A}_{2,4} & \ldots & \bar{A}_{2,2 \ell} \\
A_{4,2} & \bar{A}_{4,4} & \ldots & \bar{A}_{4,2 \ell} \\
\vdots & \ddots & \ddots & \vdots \\
O & O & A_{2 \ell, 2 \ell-2} & A_{2 \ell, 2 \ell}
\end{array}\right]
\end{aligned}
$$

It follows by inspection that the pair $\left(\breve{A}_{1}, \breve{B}_{1}\right)$ is controllable. Thus, by the above choice of $F$, we made $\sum_{i=1}^{\ell} \nu_{2}^{(i)}$ eigenvalues of $A+B_{2} F$ uncontrollable via $B_{1}$, additionally to the $n-r$ uncontrollable original eigenvalues. The first $n_{c}=\sum_{i=1}^{\ell} \nu_{1}^{(i)}$ columns $V_{1}$ of $V$ satisfy

$$
A V_{1}=V_{1} \breve{A}_{1}-B_{1} F V_{1}, \quad B_{1}=V_{1} \breve{B}_{1}
$$

and thus span a Type $I$ dynamic cover of dimension $n_{c}$ for the pair $\left(A,\left[\begin{array}{ll}B_{2} & B_{1}\end{array}\right]\right)$. The following result can be shown using the results of [4]:

Theorem 2: The Type I dynamic cover $\mathcal{V}=\operatorname{span} V_{1}$ has minimum dimension. 


\section{NUMERICAL ASPECTS}

The key reduction of system matrices to the special controllability form can be performed by using exclusively orthogonal similarity transformations. It can be shown that the computed condensed matrices $\widehat{A}$ and $\widehat{B}$ are exact for matrices which are nearby to the original matrices $A$ and $B$, respectively. Thus this part of the reduction is numerically backward stable. In implementing the algorithm, the row compressions are usually performed using rank revealing QR-factorizations with column pivoting [3]. To make rank determinations even more reliable, QR-decompositions and singular value decompositions can be combined (see [9]).

The rank revealing QR-decomposition is performed by employing Householder transformations, and these transformations are immediately applied to $B, A$ and $Z$, without accumulating them in $U_{1}$ and $U_{2}$. Thus, the reduction is essentially the same as that required to compute the Hessenberg form of the matrix $A$, which amounts to about $7 / 3 n^{3}$ floating-point operations (flops). Note that for solving the problem (1), the accumulation of $Z$ is not even necessary, since all right transformations can be directly applied to $C$.

The computations at Stage II to determine a basis for the minimal dynamic cover and the computation of feadback/feedforward matrices involve the solution of many, generally overdetermined, linear equations. For the computation of the basis for $\mathcal{V}$, we can estimate the condition numbers of the overall transformation matrix by computing $\|V\|_{F}^{2}=\|U\|_{F}^{2}$. If this norm is relatively small (e.g., $\|V\|_{F}^{2} \leq 10000$ ) then practically there is no danger for a significant loss of accuracy due to nonorthogonal reduction. Note that it is very important to compute these condition numbers, since large values of them provide a clear hint of possible accuracy losses. In practice, it suffices to look at the largest magnitudes of elements of $U$ used at Stage II to obtain equivalent information. For the computation of the feedback/feedforward matrices, condition numbers for solving the underlying equations can be also easily estimated. For the Stage II reduction, a simple operation count is possible by assuming all blocks $1 \times 1$ and this amounts to about $n^{3} / 4$ flops.

\section{MINIMUM COVERS WITH STABILIZATION}

In some applications it is important to achieve simultaneously that the resulting feedback is stabilizing. For a Type II cover, this amounts to determine $F, G$ and $V$ such that the resulting $\breve{A}_{1}$ has all eigenvalues in an appropriate stability domain $\mathbf{C}^{-}$. This goal can not always be achieved, but it is always possible to move a maximum number of eigenvalues in this domain. To show how this is possible, consider the matrix pair $\left(P^{T} \widetilde{A} P, P^{T} \widetilde{B}\right)$, where $\widetilde{A}$ and $\widetilde{B}$ are the resulting matrices at the end of Stage II and $P^{T}$ is the permutation matrix (3). The matrices of this pair have the form

$$
\begin{gathered}
P^{T} \widetilde{B}=\left[\begin{array}{cc}
O & \widetilde{B}_{12} \\
\widetilde{B}_{21} & \widetilde{B}_{22} \\
O & O \\
\hline O & O
\end{array}\right] \\
P^{T} \widetilde{A} P=\left[\begin{array}{ccc|c}
\widetilde{A}_{11} & \widetilde{A}_{12} & \widetilde{A}_{13} & * \\
\widetilde{A}_{21} & \widetilde{A}_{22} & \widetilde{A}_{23} & * \\
O & \widetilde{A}_{32} & \widetilde{A}_{33} & * \\
\hline O & O & O & A_{\bar{c}}
\end{array}\right]
\end{gathered}
$$

where the pair $\left(\widetilde{A}_{11}, \widetilde{B}_{12}\right)$ is controllable, and $\widetilde{B}_{21}$ and has full row rank. Note that the Stage II special reduction achieves basically to zero the block $\widetilde{A}_{31}$, while the feedback matrix $F$ and feedforward matrix $G$ achieve additionally to zero $\widetilde{A}_{21}$ and $\widetilde{B}_{22}$, respectively, by exploiting the full rank property of $\widetilde{B}_{21}$.

Consider the transformation matrix

$$
T=\left[\begin{array}{ccc|c}
I & O & O & O \\
X & I & O & O \\
O & O & I & O \\
\hline O & O & O & I
\end{array}\right]
$$

partitioned in accordance with the structure of $P^{T} \widetilde{A} P$. It follows that

$$
\begin{gathered}
T^{-1} P^{T} \widetilde{B}=\left[\begin{array}{cc}
O & \widetilde{B}_{12} \\
\widetilde{B}_{21} & \bar{B}_{22} \\
O & O \\
\hline O & O
\end{array}\right] \\
T^{-1} P^{T} \widetilde{A} P T=\left[\begin{array}{ccc|c}
\widetilde{A}_{11}+\widetilde{A}_{12} X & \widetilde{A}_{12} & \widetilde{A}_{13} & * \\
\bar{A}_{21} & \bar{A}_{22} & \bar{A}_{23} & * \\
\widetilde{A}_{32} X & \widetilde{A}_{32} & \widetilde{A}_{33} & * \\
\hline O & O & O & A_{\bar{c}}
\end{array}\right]
\end{gathered}
$$

where we denoted with bars the changed quantities. If we choose $X$ such that $\widetilde{A}_{32} X=0$, we can preserve the structure of the original pair $\left(P^{T} \widetilde{A} P, P^{T} \widetilde{B}\right)$. Thus, defining $V$ as $V=Z U P T$, we can compute the feedback and feedforward matrices $F$ and $G$ exactly as before.

With $T$ chosen as above, the resulting $\breve{A}_{1}$ is $\widetilde{A}_{11}+\widetilde{A}_{12} X$ and we can try to exploit this parametric freedom to move the eigenvalues of $\widetilde{A}_{11}$ to stable locations. The following straightforward computations are necessary for this purpose:

1) Compute $X_{N}$ with orthonormal columns such that span $X_{N}$ is the right nullspace of $\widetilde{A}_{32}$.

2) Compute $\widetilde{F}$ to place a maximum number of eigenvalues of $\widetilde{A}_{11}+\widetilde{A}_{12} X_{N} \widetilde{F}$ into the stability domain $\mathbb{C}^{-}$.

3) Define $X=X_{N} \widetilde{F}$.

All steps of this algorithms can be performed using numerically reliable computations. The computation of $X_{N}$ is straightforward, since $\widetilde{A}_{32}$ is part of a staircase form. Thus, no further rank determination is necessary and $X_{N}$ results from an RQ-like decomposition of $\widetilde{A}_{32}$ which exploits the 
full row rank of its leading nonzero rows. To determine $\widetilde{F}$, the most appropriate method is to apply a partial pole assignment technique like that of [10]. This approach can easily accommodate with non-stabilizable pairs, by moving only the controllable unstable eigenvalues of $\widetilde{A}_{11}$ into $\mathbf{C}^{-}$. If the pair $\left(\widetilde{A}_{11}, \widetilde{A}_{12} X_{N}\right)$ is stabilizable then this algorithm can assign all unstable eigenvalues to arbitrary stable locations using minimum norm local feedbacks. In this way, the norm of $X$ is minimized as well and thus also the condition number of the transformation matrix $T$. A similar approach can be devised for determining Type I minimal covers with stabilization.

A specific aspect of determining minimal dynamic covers is the non-uniqueness of the resulting solution triple $(F, G, V)$. This non-uniqueness manifests at several points of the proposed approach and can have negative or positive influence on the stabilizability properties determined by the triple $\left(\widetilde{A}_{11}, \widetilde{A}_{12}, \widetilde{A}_{32}\right)$. For example, selecting differently at Stage I the linearly independent columns in $B_{1}^{(j-1)}$ and $B_{2}^{(j-1)}$ or computing differently the blocks of $U$ at Stage II when solving the underdetermined linear systems can lead to different minimal covers and different stabilizability properties. For numerical implementations, we recommend those solutions which ensure the best numerical properties of the proposed approach (e.g., selecting independent columns using column pivoting or determining least-norm solutions of all underdetermined linear systems).

\section{CONCLUSIONS}

We proposed efficient algorithms to compute two types of minimal dynamic covers, which have many important applications in various structural synthesis problems of linear systems. The proposed algorithms rely on the extensive use of orthogonal transformations. The use of non-orthogonal transformations at the final step of the reduction process allows also to obtain a precise estimation of possible accuracy losses induced by the overall reduction. Thus the proposed algorithm, although not numerically stable, can be considered numerically reliable.

The proposed approach has been extended recently [15] to generalized system representations, relying on the orthogonal staircase algorithm for descriptor systems proposed in [11]. It is certainly possible to extended our approach to periodic systems as well along the lines of the recently proposed periodic staircase algorithm in [12].

The Stage I algorithms for both standard and descriptor systems [15] has been implemented in Fortran 77 and can be used via a mex-file interface from MATLAB. Furthermore, the Stage II of the proposed approach has been implemented in MATLAB and underlies the implementation of methods to compute least order left or right inverses and least order solutions of linear rational equations. All this software is part of the Descriptor Systems Toolbox for MATLAB ${ }^{1}$.

\footnotetext{
${ }^{1}$ http://www.robotic.dlr.de/control/num/desctool.html
}

\section{REFERENCES}

[1] E. Emre and L. M. Silverman. Minimal dynamic inverses for linear systems with arbitrary initial states. IEEE Trans. Autom. Control, 21:766 - 769, 1976.

[2] E. Emre, L. M. Silverman, and K. Glover. Generalized dynamic covers for linear systems with applications to deterministic identification and realization problems. IEEE Trans. Autom. Control, 22:26 - 35, 1977.

[3] G. H. Golub and C. F. Van Loan. Matrix Computations. John Hopkins University Press, Baltimore, 1989.

[4] G. Kimura. Geometric structure of observers for linear feedback control laws. IEEE Trans. Autom. Control, 22:846-855, 1977.

[5] V. Klema and A. Laub. The singular value decomposition: Its computation and some applications. IEEE Trans. Autom. Control, 25:164 - 176, 1980.

[6] A. S. Morse. Minimal solutions to transfer matrix equations. IEEE Trans. Autom. Control, 21:131-133, 1976.

[7] C. C. Paige. Properties of numerical algorithms related to computing controllability. IEEE Trans. Autom. Control, 26:130 - 138, 1981.

[8] P. Van Dooren. The generalized eigenstructure problem in linear systems theory. IEEE Trans. Autom. Control, 26:111-129, 1981.

[9] A. Varga. Numerically stable algorithm for standard controllability form determination. Electron. Lett., 17:74-75, 1981.

[10] A. Varga. A Schur method for pole assignment. IEEE Trans. Autom. Control, 26:517-519, 1981.

[11] A. Varga. Computation of irreducible generalized statespace realizations. Kybernetika, 26:89-106, 1990.

[12] A. Varga. Computation of Kalman decompositions of periodic systems. Proc. of ECC'2003, Cambridge, UK, 2003.

[13] A. Varga. New computational approach for the design of faul detection and isolation filters. In M. Voicu (Ed.), Advances in Automatic Control, pp. 367-381, Kluwer Academic Publishers, 2003.

[14] A. Varga. On computing least order fault detectors using rational nullspace bases. Prepr. of IFAC Symp. SAFEPROCESS'2003, Washington D.C., 2003.

[15] A. Varga. Reliable algorithms for computing minimal dynamic covers for descriptor systems (in preparation), 2003.

[16] W. M. Wonham. Linear Multivariable Control: a Geometric Approach. Springer Verlag, New York, 1979.

[17] W. M. Wonham and A. S. Morse. Feedback invariants of linear multivariable systems. Automatica, 8:93-100, 1972. 